\title{
Effectiveness of silicone-based resilient denture liners on the patient-reported chewing ability: A randomized controlled trial
}

\author{
Yoshiteru Furuya a , Suguru Kimoto ${ }^{c,}$, Nobuhiko Furuse ${ }^{b}$, So Furokawa ${ }^{b}$, Kentaro Igarashi ${ }^{b}$, \\ Asako Suzuki ${ }^{b}$, Yasuhiko Kawai ${ }^{b}$ \\ a Nihon University Graduate School of Dentistry at Matsudo, Removable Prosthodontics and Geriatric Oral Health, Matsudo, Japan \\ ${ }^{b}$ Department of Removable Prosthodontics and Geriatric Oral Health, Nihon University School of Dentistry at Matsudo, Matsudo, Japan \\ ' Department of Gerodontology and Home Care Dentistry, School of Dentistry Aichi Gakuin University, Nagoya, Japan
}

\begin{abstract}
Purpose: This randomized controlled trial (RCT) aimed to elucidate the effectiveness of silicone-based resilient denture liners on perceived chewing ability.

Methods: The RCT included completely edentulous patients on both the maxilla and mandible, who were willing to fabricate a new set of complete dentures. A random permuted block method (block size: 4) was used to assign groups to receive maxilla conventional complete denture and mandibular with either a conventional complete denture (CD) or complete demure relined with silicone-based resilient denture liner (RD). The patient-reported chewing ability of six foods, soybean curd, fish sausage, soybean sprout, cubic rice cracker, hard rice cracker, and dry squid, on a 100-mm visual analog scale was measured and reported at the final adjustment and three months after the final adjustment. Mann-Whitney $U$ test was used to analyze the differences between the $\mathrm{CD}$ and $\mathrm{RD}(p<0.05)$.

Results: Edentulous patients wearing mandibular complete denture with RD had a higher patient-reported chewing ability than those wearing a CD, but the fish sausage did not in the RD three months after the final adjustment. It also took three months for the patient-reported masticatory ability to improve for cubic rice crackers, hard rice crackers, and dry squid and to show a difference between the RD and CD groups.

Conclusion: Although limited to some food types, mandibular complete dentures relined with silicone-based liners improved patient-reported chewing ability.
\end{abstract}

Keywords: Chewing ability, Patient-reported chewing ability, Randomized controlled trial, Resilient denture liner

Received 17 June 2021, Accepted 30 September 2021, Available online 30 October 2021

\section{Introduction}

A decrease in the remaining teeth relates to a declined masticatory function[1]. Removable denture treatment contributes to restoring masticatory function in tooth loss, but limitation lies in an inherent healthy dentition[2,3]. As tooth loss progress, a complete denture is used for full edentulousness. However, their masticatory function is significantly lower than that of dentate individuals, and it is presumed that the average number of masticatory functions is less than a quarter of dentate individuals[3]. The decline in masticatory function is closely related to the nutritional status of the elderly in the previous report[4]; which is assumed that malnutrition may be caused by decreased masticatory function. Low nutrition may lead to frailty and sarcopenia in the long-term process[5], and in consequence, it may be related to the healthy life expectancy and quality

DOI: https://doi.org/10.2186/jpr.JPR_D_21_00159

*Corresponding author: Suguru Kimoto, Department of Gerodontology and Home Care Dentistry, School of Dentistry Aichi Gakuin University, 2-11 Suemoritouri Chikusaku, Nagoya, Aichi 464-8651, Japan.

E-mail address: kimoto.suguru@gmail.com

Copyright: @ 2021 Japan Prosthodontic Society. All rights reserved of life of the elderly[6]. Therefore, to improve masticatory function in edentulous individuals are an essential issue.

One procedure to improve masticatory function is to apply a resilient denture liner, rather than the conventional method, to complete dentures. The flexibility, resilience, and shock absorbency of resilient denture liners offer greater versatility for edentulous patients facing eating difficulties[7]. Several outcomes have been used for edentulous patients to assess the efficacy of silicone-based resilient denture liner (RD). Hayakawa et al. reported changes in the masticatory function of complete denture wearers after relining the mandibular denture with a soft denture liner using maximum biting force, masticatory performance, and electromyography of the masseter muscle during mastication as outcomes[8]. Murata et al. evaluated masticatory function in edentulous patients with the acrylic-based $\mathrm{RD}$, silicone-based RD and tissue conditioner using measurements of maximum bite force, chewing time, and chewing frequency for test food samples as outcomes[9]. Kimoto et al. used masticatory performance measured by the sieving method with peanuts as a test food, muscle activity measured by electromyograms, maximum occlusal force measured by pressure-sensitive sheets[10], and satisfaction rating on chewing measured by $100-\mathrm{mm}$ visual analog scale 
(VAS) as outcomes in their randomized controlled trial (RCT) to verify the clinical effect of silicone-based RD on masticatory function[11]. Pisani et al. measured oral health-related quality of life in complete denture wearers with a silicone-based soft liner using masticatoryrelated complaints in Oral Health Impact Profile for Edentulous (OHIP-EDENT) as an outcome[12]. Tata et al. measured the masticatory performance through the sieving method using peanuts in complete denture wearers with silicone-based RD[13]. Pisani et al. also used muscle activity in the masseter and temporalis, measured by electromyograms as outcomes, to evaluate masticatory function in complete denture wearers with a silicone-based soft liner[14].

We believe that masticatory functions must be assessed by patient-reported outcome such as food in daily diet. To our knowledge, food intake in daily diet has not been used as an outcome for edentulous patients with a silicone-based RD. Thus, we conducted an $\mathrm{RCT}$ to evaluate the food intake of edentulous patients with RD. The purpose of this study was to investigate the effectiveness of RD on patient-reported chewing ability food intake through VAS measurements. The null hypothesis was that there were no significantly different patient-reported chewing abilities between the conventional denture (CD) and RD groups.

\section{Materials and Methods}

\subsection{Trial registration}

The RCT was registered with the University Hospital Medical Information Network (UMIN) Registry (UMIN000027601) on June 2, 2016. We carried out this study following the principles of the Declaration of Helsinki and the Consolidated Standards of Reporting Trials (CONSORT) guidelines. The study protocol was reviewed and approved by the human ethics committees of Nihon University School of Dentistry at Matsudo (IRB project number: EC19-17-001-1).

\subsection{Inclusion and exclusion criteria}

\subsubsection{Inclusion criteria}

Participants had to be edentulous in both jaws and willing to have a new set of complete dentures to be included in the study.

\subsubsection{Exclusion criteria}

Patients with diabetes mellitus, trigeminal neuralgia, postherpetic neuralgia, signs and symptoms of orofacial pain disorders, pacemakers, apparent cognitive impairment, or a history of mandibular surgery were excluded[7].

\subsection{Sample size calculation}

A between-group difference of $20 \mathrm{~mm}$ on the VAS ratings for hard rice crackers was considered a significant difference. By using a standard deviation of $20.4 \mathrm{~mm}$ for the CD group and $15.1 \mathrm{~mm}$ for the $\mathrm{RD}$ group, which were based on an interim analysis of the present study, 18 subjects per group, including an assumed dropout rate of $10 \%$, was be required to achieve $80 \%$ power with an alpha level of $5 \%$.

\subsection{Randomization and allocation concealment}

The participants provided informed consent on enrolment before randomization and were recruited consecutively between May
2017 and December 2019. A random permuted block method was used to ensure that a certain proportion of patients were allocated into each treatment[15]. Using the allocation numbers generated by the "RAND" function in Excel (Microsoft Japan Co. Ltd., Tokyo, Japan) participants were randomly assigned to either the $C D$ or the $\mathrm{RD}$ group. Blinding of the participants was not feasible, as they were aware of their denture type.

\subsection{Fabrication of dentures (Interventions)}

Impression-taking for the maxillary and mandibular complete dentures was carried out following two processes: the preliminary impression was taken using alginate impression material (Algiace $Z$, Dentsply Sirona, Tokyo, Japan) and a stock tray (Mesh tray, Hayashi Dental Supply, Tokyo, Japan), and the final impression was taken using silicone impression material (Exadenture, GC Corporation, Tokyo, Japan) with impression compound (Peri Compound, GC Corporation, Tokyo, Japan) and a border-molded custom tray. After occlusal registration, artificial teeth arrangements (Surpass, GC Corporation, Tokyo, Japan) with a fully bilateral balanced articulation occlusal scheme were carried out on the cast using a semi-adjustable articulator (Hanau H2, Teledyne Water Pik, Fort Collins, Colorado, USA) and a facebow record.

After the fitting session, the conventional dentures were fabricated with conventional heat-activated acrylic resin (Urban, Shofu Dental Corporation, Kyoto, Japan). The dentures with RD were fabricated with the same acrylic resin and a 2-mm-thick permanent silicone-based denture liner (Sofreliner MS, Tokuyama Dental Corporation, Tokyo, Japan).

\subsection{Participant characteristics}

The participants were asked about their age, sex, edentulous period, number of previous dentures, and denture difficulty class at the beginning of the trial.

\subsection{Outcome measurement}

2.7.1. Patient-reported chewing ability with a $100-\mathrm{mm}$ visual analog scale

The patient-reported chewing ability during the mastication of six foods, namely, soybean curd, fish sausage, soybean sprout, cubic rice cracker, hard rice cracker, and dry squid, were measured using the 100-mm VAS[16]. The six foods were selected based on Sato et al.'s study, which evaluated the chewing function of patients with complete denture[17]. The VAS data represented "very easy to chew" (100) at the extreme right and "very difficult to chew" (0) at the extreme left. Data was be recorded as continuous variables by measuring the distance at 1-mm intervals by using calipers, beginning at the extreme left.

\subsubsection{Schedule for measuring outcomes}

The patient-reported chewing ability was measured three times-once when the participants received the existing denture (baseline), second at the final completion of the denture adjustment (final adjustment), and third at the recalled visit (three months after the final adjustment). When the participants complained of any symptoms during the trials, e.g., discomfort or pain, and required denture adjustment, the clinicians treated the participants appropriately. 


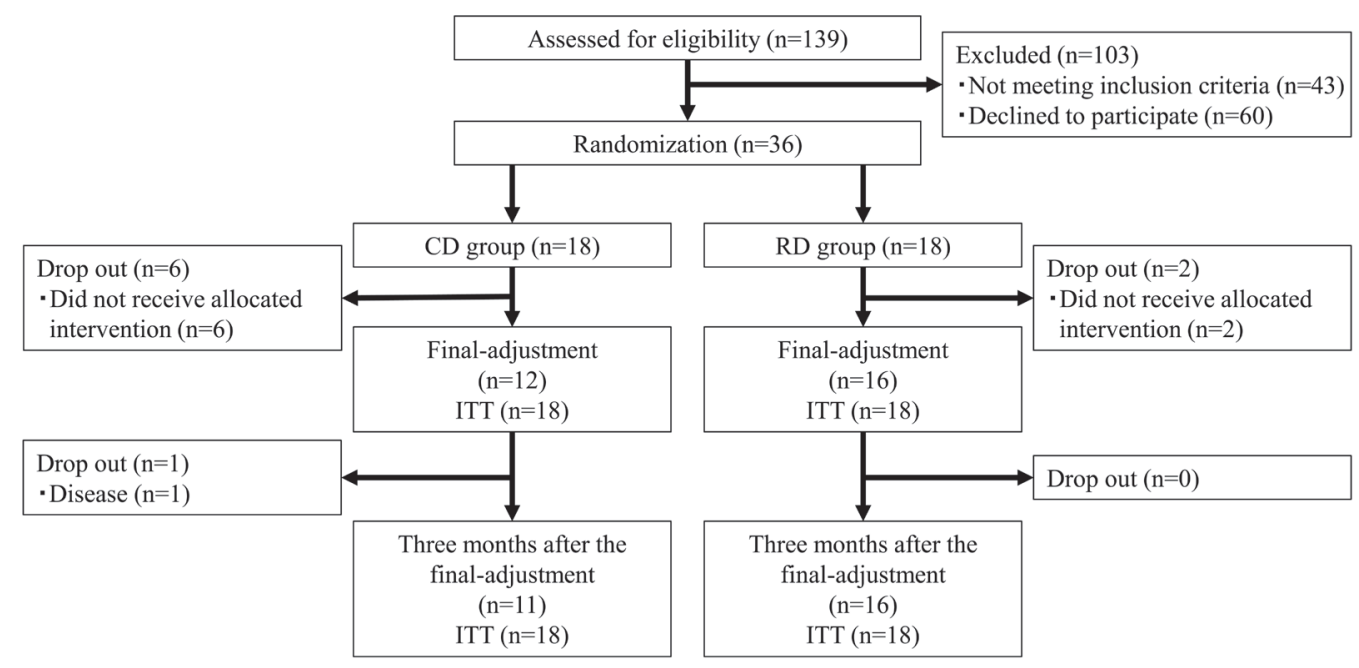

Fig. 1. Trial flowchart. The participants were consecutively recruited between May 2017 and December 2019. The follow-up rate was $75 \%$. CD, conventional denture base; RD, resilient denture liner; ITT, intention to treat analysis.

Table 1. Baseline participants characteristics

\begin{tabular}{lccc}
\hline Participants characteristics $(\mathrm{n}=36)$ & $\mathrm{CD}(\mathrm{n}=18)$ & $\mathrm{RD}(\mathrm{n}=18)$ & $\mathrm{P}$-value \\
\hline Age (years) & $73.2(7.8)$ & $77.7(7.8)$ & $0.09^{\mathrm{a}}$ \\
Sex (female/male) & $8 / 10$ & $8 / 10$ & $1.00^{\mathrm{b}}$ \\
Edentulous period (months) & $160.1(96.1)$ & $109.8(92.5)$ & $0.12^{\mathrm{a}}$ \\
\hline No. of previous dentures & & & \\
$\quad$ Maxilla & $2(1.6)$ & $1.9(1.1)$ & $0.81^{\mathrm{a}}$ \\
$\quad$ Mandible & $2(1.6)$ & $1.9(1.1)$ & $0.81^{\mathrm{a}}$ \\
Classification of denture difficulty (I,II,III,IV) & $(11,6,1,0)$ & $(10,7,1,0)$ & $0.94^{\mathrm{b}}$ \\
\hline
\end{tabular}

a: T-test, b: Chi-squared test

$\mathrm{CD}$, conventional denture base; $\mathrm{RD}$, resilient denture liner

Table 1 was same as previous report[18], because participants were same.

\subsection{Data analysis}

The intention-to-treat principle (ITT) was used for any missing data after the randomization. The Kolmogorov-Smirnov test assessed the normality of the outcome variables, that is, the patient-reported chewing abilities. As the results indicated that the data were not normally distributed, a non-parametric test, Mann-Whitney U test, was used. The analysis of the categorical variables between the two groups was carried out with a chi-square test. All statistical analyses were performed using the IBM SPSS Statistics Package v21 (IBM, Armonk, New York, USA). The statistical significance was set at $5 \%$.

\section{Results}

\subsection{Participant flow}

The trial flowchart of the subjects who participated in this study is shown in Figure 1. Twenty-five percent of participants were dropped out during the trial. The intention to treat analyses was carried out.

\subsection{Participant characteristics}

The participants' characteristics at baseline measurement had no significant difference between the RD and CD groups (Table 1).
The participants in this study were same as those in the previous report[18].

\subsection{Patient-reported chewing ability}

Table 2 shows the median values (lower quartile, upper quartile) for each question for the patient-reported chewing ability in each group at the final adjustment and three months later. The patientreported chewing ability at baseline showed no significant difference ( $p>0.05$ ) between the RD and CD groups in all foods; however, at the final adjustment, significant differences between the RD and CD groups in the soybean curd ( $p=0.044)$, fish sausage $(p=0.033)$, and soybean sprout ( $p=0.023$ ) was observed. At three months after the final adjustment, significant differences between the RD and CD groups in the soybean curd ( $p=0.019)$, soybean sprout $(p=0.049)$, cubic rice cracker $(p=0.013)$, hard rice cracker $(p=0.020)$, and dry squid ( $p=0.049$ ) were indicated.

\section{Discussion}

This randomized controlled trial revealed that edentulous patients wearing mandibular complete denture with RD had a higher patient-reported chewing ability than those without RD. There were three patterns of patient-reported chewing ability obtained from VAS for six foods in the RD compared to the CD group as follows: (1) 
Table 2. Median values (lower quartile, upper quartile) of the patient-reported chewing ability with a $100 \mathrm{~mm}$ visual analog scale in the RD group and CD group

\begin{tabular}{|c|c|c|c|c|c|c|c|c|c|c|c|c|c|c|c|}
\hline \multirow[b]{3}{*}{ Soybean curd } & \multicolumn{5}{|c|}{ Base line } & \multicolumn{5}{|c|}{ Final-adjustment } & \multicolumn{5}{|c|}{ Three months after the final-adjustment } \\
\hline & \multicolumn{2}{|c|}{$C D(n=18)$} & \multicolumn{2}{|c|}{$\mathrm{RD}(\mathrm{n}=18)$} & \multirow{2}{*}{$\frac{\text { P-value }}{0.744}$} & \multicolumn{2}{|r|}{$C D(n=18)$} & \multicolumn{2}{|c|}{$\mathrm{RD}(\mathrm{n}=18)$} & \multirow{2}{*}{$\frac{P \text {-value }}{0.044^{*}}$} & \multicolumn{2}{|c|}{$C D(n=18)$} & \multicolumn{2}{|c|}{$\mathrm{RD}(\mathrm{n}=18)$} & \multirow{2}{*}{$\frac{P \text {-value }}{0.019^{*}}$} \\
\hline & 93 & $(87.5-100)$ & 92 & $(87.5-100)$ & & 93 & $(92.8-100)$ & 100 & $(97-100)$ & & 94 & $(94-100)$ & 100 & $(99.5-100)$ & \\
\hline Fish sausage & 86 & $(77-100)$ & 74 & $(50-100)$ & 0.221 & 89 & $(89-100)$ & 100 & $(92-100)$ & $0.033^{*}$ & 93 & $(93-100)$ & 100 & $(92.8-100)$ & 0.146 \\
\hline Soybean sprout & 70 & $(49.8-83.5)$ & 79 & $(54.3-100)$ & 0.179 & 90 & $(89-100)$ & 100 & $(94.8-100)$ & $0.023^{*}$ & 91.5 & $(91-100)$ & 100 & $(94-100)$ & $0.049^{*}$ \\
\hline Cubic rice cracker & 67 & $(40-95.5)$ & 62 & $(26.3-100)$ & 0.774 & 82 & $(81.5-94)$ & 98.5 & $(80.8-100)$ & 0.123 & 83 & $(81-100)$ & 100 & $(93.3-100)$ & $0.013^{*}$ \\
\hline Hard rice cracker & 58 & $(26.5-89.3)$ & 60 & $(18.3-94.8)$ & 0.634 & 77 & (75.8-91.3) & 97.5 & $(65.8-100)$ & 0.191 & 81 & (78.8-94.8) & 100 & $(87-100)$ & $0.020^{*}$ \\
\hline Dry squid & 58 & $(17-89)$ & 36 & $(0-59.8)$ & 0.181 & 60 & $(46.3-85.5)$ & 75 & $(23.5-100)$ & 0.258 & 68 & $(68-89.5)$ & 93.5 & $(75.5-100)$ & $0.049^{*}$ \\
\hline
\end{tabular}

Mann-Whitney U Test

$C D$, conventional denture base; $R D$, resilient denture liner

* Significant difference $(p<0.05)$

higher at three months after the final adjustment, (2) higher at immediately after final adjustment, and (3) higher at the final adjustment, but with no difference at three months after the final adjustment.

The first pattern was observed in the cubic rice cracker, hard rice cracker, and dry squid. The patient-reported chewing ability obtained from the three foods in the RD group showed higher values than the CD group three months after the final adjustment. Considering three types of foods that were hard and difficult to eat, the reason for superiority in the RD group could be explained by its elasticity. It is well-known that these three foods are very hard to masticate for complete denture wearers[19]. Denture wearers have only one-fifth to one-fourth of the bite force of a natural dentition[3,20] because they cannot bite more than the pain threshold of the alveolar ridge mucosa. However, Yamamoto et al. reported in their simulated study that silicone-based $\mathrm{RD}$ reduces the maximum stress on the artificial mucosa by almost $16.2 \%$ compared to the CD-based material used for the CD group in this study[20]. This cushioning effect of the RD contributed to the difference in the patient-reported chewing abilities between the RD and CD groups. Furthermore, Furokawa et al. showed that edentulous patients wearing mandibular complete dentures with silicone-based RDs had an increased pain threshold[7]. Therefore, the greater patient-reported chewing ability in the $\mathrm{RD}$ group compared to the CD group in the present study may have been attributed to the mixed effect of RD on the alveolar ridge: elasticity of the liner to reduce the stress on the alveolar ridge mucosa and to the increase pain threshold.

Immediately after the final adjustment, the patient-reported chewing ability on the three foods in the RD group was not higher than that in the CD group; that is, the patient-reported chewing ability on the three foods improved only in the RD group and not in the CD group. Kimoto et al. reported that edentulous patients wearing complete mandibular dentures with RD showed better masticatory performance than that with $C D$ three months after the completion of the denture adjustment; however, this did not show one month after the completion of denture adjustment[10]. Although there was a difference between the subjective and objective evaluations, the differences in the evaluation between the complete denture wearers in the CD and RD groups over time were almost the same. These differences in the patient-reported chewing ability over time might be explained by the fact that edentulous patients in the RD group gradually obtained the necessary skills for mastication by repeating a daily practice of food mastication. Furthermore, we instructed participants to consume soft foods after receiving their new dentures. Therefore, it was possible that the early period after the completion of the new denture adjustment was insufficient for the adaptation period to the denture, and the participants could not fully use the viscoelastic properties of the RD.

The second pattern was observed in the soybean curd and soybean sprout. The RD group had a higher patient-reported chewing ability for the soybean curd and soybean sprout than that in the CD group immediately after the final adjustment. We believed that the pattern of patient-reported chewing ability obtained from these two foods were due to different reasons. It was difficult to say that the reason for the differences between the RD and CD groups could be derived from the cushion effect of the RD on the alveolar ridge to relieve pain, given that soybean curd can easily be chewed, such that participants can squash the pudding with their tongue without pain. Koshino et al. classified the pudding into grade 1 difficulty, pertaining to foods that are most easily chewed[19]. The soybean curd is a kind of Japanese pudding. Thus, the results on the patient-reported chewing ability might be derived from participants' feeling of a denture, with or without an RD. Furokawa et al. reported that the psychological discomfort when wearing a denture was significantly lower in the RD group than that in the CD group[7]. The emotional effect derived from silicone-based RD would increase the patient-reported chewing ability on the soybean curd in the RD group and induce the difference of patient-reported chewing ability on the soybean curd between the RD and CD groups. In contrast, soybean sprout is harder than soybean curd; thus, an increased bite force might be necessary to break down the soybean sprout more, compressing the mandibular alveolar ride mucosa and, consequently, inducing pain. We believed that the RD group might increase the patient-reported chewing ability on the soybean sprout under the cushion effect of the RD, and the difference between the RD and CD groups might be observed.

The third pattern was observed in the fish sausage. Even though the difference was observed for the fish sausage immediately after the final adjustment, the difference was not observed three months after the final adjustment. The fish sausage is made by cooking fish meat with heat, and cooked fish meat increases elasticity. We believed that the elastic physical properties of food might contribute to the patient-reported chewing ability, given that RD itself is elastic.

The similarity of the baseline characteristics between the two groups showed the good randomization to contribute the high internal validity of data and the control of any bias to potentially modify the data. However, there were several limitations to this RCT. First, the current study was conducted at a single institution in Japan, 
which indicates low external validity. Second, it could not be completely blinded because the surgeons were able to recognize the differences between the groups by checking the completed dentures. In addition, a total of nine dropouts were observed, seven in the CD group and two in the RD group; thus, a complete follow-up could not be performed. Furthermore, clinicians would have clinical questions such as how the chewing ability change with time of resilient liner deterioration and how the acrylic-based resilient denture liners not used in this study affect the chewing ability of patients with complete denture. Considering the limitations and clinical questions, further studies, including multiple research centers in Japan and larger sample sizes, are needed to confirm the results of this study.

\section{Conclusion}

This study concludes that a silicone-based elastic denture liner improved patient-reported chewing ability against most foods compared to CD-based materials.

\section{Acknowledgements}

This research was carried out with support from the Japan Society for the Promotion of Science, Grant/Award Number: Scientific Research (C)2\#17K11767.

\section{Conflicts of interest}

The authors declare that they have no known competing financial interests or personal relationships that could have appeared to influence the work reported in this paper.

\section{References}

[1] Ikebe K, Matsuda K, Kagawa R, Enoki K, Yoshida M, Maeda Y, et al. Association of masticatory performance with age, gender, number of teeth, occlusal force and salivary flow in Japanese older adults: is ageing a risk factor for masticatory dysfunction? Arch Oral Biol. 2011;56:991-6. https:// doi.org/10.1016/j.archoralbio.2011.03.019, PMID:21529776

[2] Manly RS, Braley LC. Masticatory performance and efficiency. J Dent Res. 1950;29:448-62. https://doi.org/10.1177/00220345500290040701, PMID:15436916

[3] Kapur KK, Soman SD. Masticatory performance and efficiency in denture wearers. J Prosthet Dent. 1964;14:687-94. https://doi.org/10.1016/00223913(64)90203-3

[4] Motokawa K, Mikami Y, Shirobe M, Edahiro A, Ohara Y, Iwasaki M, et al. Relationship between Chewing Ability and Nutritional Status in Japanese Older Adults: A Cross-Sectional Study. Int J Environ Res Public Health. 2021;18:1216. https://doi.org/10.3390/ijerph18031216, PMID:33572969

[5] Wayler AH, Muench ME, Kapur KK, Chauncey HH. Masticatory performance and food acceptability in persons with removable partial dentures, full dentures and intact natural dentition. J Gerontol. 1984;39:284-9. https:// doi.org/10.1093/geronj/39.3.284, PMID:6715804
[6] Yurkstas AA, Emerson WH. Decreased masticatory function in denture patients. J Prosthet Dent. 1964;14:931-4. https://doi.org/10.1016/00223913(64)90021-6

[7] Furokawa S, Kimoto S, Furuse N, Furuya Y, Ogawa T, Nakashima Y, et al. The effects of silicone-based resilient denture liners on pain: A randomized controlled trial. J Prosthodont Res. 2020;64:417-23. https://doi.org/10.1016/j. jpor.2019.11.006, PMID:32061571

[8] Hayakawa I, Hirano S, Takahashi Y, Keh ES. Changes in the masticatory function of complete denture wearers after relining the mandibular denture with a soft denture liner. Int J Prosthodont. 2000;13:227-31. PMID:11203637

[9] Murata H, Taguchi N, Hamada T, Kawamura M, McCabe JF. Dynamic viscoelasticity of soft liners and masticatory function. J Dent Res. 2002;81:123-8. https://doi.org/10.1177/0810123, PMID:11827257

[10] Kimoto S, So K, Yamamoto S, Ohno Y, Shinomiya M, Ogura K, et al. Randomized controlled clinical trial for verifying the effect of silicone-based resilient denture liner on the masticatory function of complete denture wearers. Int J Prosthodont. 2006;19:593-600. PMID:17165299

[11] Kimoto S, Kitamura M, Kodaira M, Yamamoto S, Ohno Y, Kawai Y, et al. Randomized controlled clinical trial on satisfaction with resilient denture liners among edentulous patients. Int J Prosthodont. 2004;17:236-40. PMID:15119878

[12] Pisani MX, Malheiros-Segundo AL, Balbino KL, Souza RF, Paranhos HFO, Lovato da Silva $\mathrm{CH}$. Oral health related quality of life of edentulous patients after denture relining with a silicone-based soft liner. Gerodontology. 2012;29:e474-80. https://doi.org/10.1111/j.1741-2358.2011.00503.x, PMID:21696442

[13] Tata S, Nandeeshwar DB. A clinical study to evaluate and compare the masticatory performance in complete denture wearers with and without soft liners. J Contemp Dent Pract. 2012;13:787-92. https://doi.org/10.5005/ jp-journals-10024-1230, PMID:23404004

[14] Pisani MX, Segundo AL, Leite VM, de Souza RF, da Silva MA, da Silva CH. Electromyography of masticatory muscles after denture relining with soft and hard denture liners. J Oral Sci. 2013;55:217-24. https://doi.org/10.2334/ josnusd.55.217, PMID:24042588

[15] Efird J. Blocked randomization with randomly selected block sizes. Int J Environ Res Public Health. 2010;8:15-20. https://doi.org/10.3390/ijerph8010015, PMID:21318011

[16] Kawai Y, Machida T, Gunji A, Kimoto S, Kobayashi K. Reliability and Validity of the Japanese Version of the Visual Analogue Scale as an Outcome Measurement of Complete Denture Prostheses. Prosthodontic Research \& Practice 2003.

[17] Sato $Y$, Minagi $S$, Akagawa $Y$, Nagasawa T. An evaluation of chewing function of complete denture wearers. J Prosthet Dent. 1989;62:50-3. https:// doi.org/10.1016/0022-3913(89)90047-4, PMID:2746541

[18] Furuya Y, Kimoto S, Furuse N, Igarashi K, Furokawa S, Kawai Y. Effectiveness of silicone-based resilient denture liners on masticatory function: A randomised controlled trial. J Dent. 2021;109:103657. https://doi.org/10.1016/j. jdent.2021.103657, PMID:33831504

[19] Koshino H, Hirai T, Toyoshita Y, Yokoyama Y, Tanaka M, Iwasaki K, et al. Development of New Food Intake Questionnaire Method for Evaluating the Ability of Mastication in Complete Denture Wearers. Prosthodontic Research \& Practice. 2008;7:12-8. https://doi.org/10.2186/prp.7.12

[20] Yamamoto S, Kimoto S, Saeki H, So K, Shinomiya M, Kobayashi K. In Vitro Study on Changes in the Stress Behavior under Simulated Mucosa Exposed to Denture Bases with Different Resilient Denture Liners. Annals of Japan Prosthodontic Society. 2009;1:277-83. https://doi.org/10.2186/ajps.1.277 